\title{
Comparison Study of Efficacyinvolving Intravitreal Ranibizumab Versus Standard Laser Mono Therapy in Patients with Visual Impairment Due To Diabetic Macula Edema.
}

\author{
Dr. Rahul Vaswani M.B,B.S, Dr. Vishal Agrawal MD, \\ Dr. Kamlesh Khilnani MS, Dr. Manisha Kalher M.B,B.S \\ (Upgraded Department Of Ophthalmology,SMS Medical College,Jaipur, India) \\ Corresponding author: Dr. Vishal Agrawal
}

\begin{abstract}
Purpose: To compare efficacy of intravitreal Ranibizumab (ivR) and standard laser in patients with visual impairment due to diabetic macular edema $(D M E)$ in terms of best corrected visual acuity(BCVA) and central macular thickness(CMT)

Design:Single blind hospital based comparative type of randomized follow-up study.:

Methods: A total of 80 eyes of 80 patients with centre-involving CSME (both diffuse and focal) were included.Subjects were subjected to treatment with either ivR(3 consecutive monthsand PRN dosing thereafter or modified macular laser therapy (MLT). Treatment outcomes in terms of BCVA and reduction in CMT were measured.

Results: There was a statistically significant change in the BCVA compared to baseline in both the groups.The average gain in visual acuity was higher in the ivRgroup.(8.825 ETDRS letters compared with 3.09ETDRS letters in the laser only group at 12 months of follow up.The decrease from the average baseline CMT was higher in the intravitreal antiVEGF group (74.22microns compared with 28.54 microns in the laser only group at
\end{abstract}

Conclusion: Both treatment modalities are effective in treatment of DME. Intravitreal Ranibizumab showed greater efficacy both in terms of final BCVA and reduction in CMT.

Financial Disclosure(s): The author(s) have no proprietary or commercial interest in any products discussed in this article.

Keywords: Intravitreal,Laser,MacularThickness, Randomization,Ranibizumab

\section{Introduction}

Diabetic macular edema (DME) in diabetic retinopathy is a leading cause of visual impairment in patients $^{1,2,4}$.The Chennai Urban Rural Epidemiological Study (CURES) was the first population based study in India (2001), which used four-field stereo retinal photographs ${ }^{5}$.The photographs were graded against standard photographs of the Early Treatment Diabetic Retinopathy Study (ETDRS) grading system for severity of retinopathy.They reported that the overall prevalence of diabetic retinopathy (DR)was $17.6 \%$ among the 1715 diabetic subjects. From 1990-2010, DR ranked as the fifth most common cause of preventable blindness and fifth most common cause of moderate to severe visual impairment

In 2010, of an estimated 285 million people worldwide with diabetes, over one-third have signs of DR, and a third of these are afflicted with vision-threatening diabetic retinopathy (VTDR), defined as severe nonproliferative DR or proliferative DR (PDR) or the presence ofDME. A review conducted in2012 suggested that up to $7 \%$ of people with diabetes may have DME and may rise to $5.4 \%$ by the year 2025 . It is higher in developed than in developing countries. The number of adults with diabetes in the world is estimated to increase by $122 \%$ (from 135 million in 1995 to 300 million in 2025). Laser therapy had been long a gold standard therapy for DME. Anti vascular endothelial growth factor (Anti VEGF) are now commonly used in combination with laser or as monotherapy in DR as level of VEGF is increased in these patients ${ }^{6}$.There are very few randomised trials comparing laser versus anti VEGF mono therapy in the treatment of diabetic macular edema.

\section{Aims And Objectives}

This proposed study is carried out to compare efficacy of intravitreal anti-VEGF (Ranibizumab) monotherapyand standard laser in patients with visual impairment due to DME in terms of:

1. Best corrected visual acuity(BCVA)

2. Central macular thickness(CMT) 


\section{Material And Methods}

Venue Of Study-The study was conducted in the Department of Ophthalmology, SMS Medical College, Jaipur, India

Study Duration-The study was conducted over a period of 12 months from $1^{\text {st }}$ June 2015 to $31^{\text {st }}$ May 2016

Sample Size-Sample size is calculated to be 40 subjects in each group at alpha error 0.05 \&power $80 \%$.

Study Design-Single blind hospital based comparative type of randomized follow-up study.

Sampling Technique: Simple Randomization technique: chit box method

Study Universe: All Patients with visual impairment due to diabetic macular edema both diffuse or focal attending department of Ophthalmology,SMS Hospital, Jaipur for treatment.

\section{Inclusion criteria:}

1. Patients with stable medication for the management of diabetes within 3 months

2. Patients having visual impairment due to focal or diffuse DME in at least 1 eye

3. Centre-involving CSME with CMT on optical coherence tomography (OCT) of $>270$ micron

4. Patients with decreased vision due to DME and not other causes

5. Those who has given written informed consent for the study

\section{Exclusion criteria:}

1. Evidence of macular ischaemia

2. Concomitant conditions in the study eye that could prevent the improvement in VA

3. Active intraocular inflammation or infection in either eye

4. Uncontrolled glaucoma in either eye

5. Treatment with anti angiogenic drugs in the study eye within 3 months

6. H/o stroke

7. Systolic blood pressure (BP) $160 \mathrm{mmHg}$ or diastolic BP $100 \mathrm{mmHg}$, untreated hypertension, or change in antihypertensive treatment

\section{Study plan of action:}

All subjects underwent a complete ophthalmic examination, including measurement of-

- BCVA (logarithm of minimal angle of resolution notation)

- Intraocular pressure,

- Fundus biomicroscopy

- Fundus fluorescein angiography and OCT was be performed for all eyes at baseline.

- In the OCT examination, six radial scans intersecting at the fovea are performed in rapid sequence. The OCT provides the average thickness as mean $6 \mathrm{SD}$ in the central macular region, centred on the patient's fovea, where the six scans intersect. The extent of macular edema was determined based on the fluorescein angiography and OCT studies.

\section{Intravitreal Ranibizumab Group}

- Intravitreal Ranibizumabwas delivered in the surgical theatre under complete aseptic conditions, using topical anesthesia.

- After painting and draping, 0.5 mgdrug was injected into the vitreous cavity using a 30-gauge needle inserted through the inferotemporal pars plana $4 \mathrm{~mm}$ from the limbus for phakic patients, $3.5 \mathrm{~mm}$ for aphakic and pseudophakic patients.

- After withdrawal of the needle, sterile cotton tipped applicator was used to apply pressure over the injection site.

- Post injection the patient was instructed to instill topical moxifloxacin ophthalmic solution $0.5 \%$,to be applied 6 times daily for 5 days

- Patients received 3 initial consecutive monthly injections of Ranibizumab (months 0-2; treatment initiation phase), followed by further treatment according to protocol-defined retreatment criteria between and including months 3 and 11 .

\section{Laser Treatment}

Modified grid laser photocoagulation.-Grid laser was performed with frequency doubled yag laser delivering 2 to 3 rows of $50 \mu \mathrm{m}$ spots, 2 visible burn width apart with burn duration 0.05 to $0.1 \mathrm{sec}$. 
Follow Up

Laser Arm: All patients in the laser arm underwent macular laser therapy(MLT) at their baseline visit or within 7 days of randomisation.

Subjects were subsequently reviewed every 4 months (16 weeks, 32 weeks, and 48 weeks), with an end of year 1 visit at 52 weeks. Retreatment (16-, 32-, and 48-week time points) was performed if clinically indicated by ETDRS guidelines. At each follow-up study visit, the investigator assessed whether persistent, recurrent or new DME was present that warranted additional photocoagulation. In general, retreatment was to be administered unless the DME had resolved or there was substantial improvement in the DME in the opinion of the investigator (e.g., > 50\% decrease in total macular thickened area or $>50 \%$ decrease in retinal thickening by OCT in central or inner subfields with previous retinal thickening). For the modified-ETDRS group, retreatment consisted of the same modified-ETDRS treatment technique. For the MLT group, the first retreatment used the same MLT technique limited to only the area of retinal thickening. If required, a second retreatment used the modified-ETDRS technique (which allows focal treatment of leaking microaneurysms in the area of retinal thickening).

Anti-VEGF Arm: All patients in the intravitreal Ranibizumab (ivR) arm underwent an injection at their baseline visit or within 7 days of randomization $(0.5 \mathrm{mg}$ in $0.05 \mathrm{ml})$. Subjects were subsequently reviewed every 6 weeks $(6,12,18,24,30,36,42$, and 48 weeks), with an end of year 1 visit at 52 weeks. After baseline ivR, patients received 2 further ivR injections (6- and 12-week time points). Subsequent ivR injections were guided by an OCT-based retreatment protocol.

In brief, if the thinnest recorded CMT was less than 270 um at 18 weeks, then treatment was continued only if macular thickness was not "stable." If CMT was greater than 270 microns at 18 weeks and subsequent visits, then ivR injections were administered until a "stable" macular thickness was attained. "Stable macular thickness" was defined as 3 consecutive visits with the CMT within 20 um of the patient's thinnest recorded CMT. Patients could thereby receive a minimum of 3 injections and a maximum of 9 injections in the first 12 months.

At each visit, a full history was taken, ETDRS BCVA was recorded by a clinical investigator, and a complete ocular examination (including anterior chamber reaction, IOP, and dilated fundoscopy) and OCT (CMT and RNFL) were performed.

\section{Outcome Measures}

1. Best corrected visual acuity (BCVA)

2. Central macular thickness (CMT)

\section{Outcome Analysis:}

- Quantitative data will be assessed in mean +/- SD

- Qualitative data will be assessed in percentage and proportions

- $\quad$ Significance of difference in means will be inferred by unpaired ' $t$ ' test.

- Significance of difference in proportions will be inferred by chi-square test.

- For significance P value equal to or less than 0.05 will be considered significant.

\section{Results}

Effect on Visual Acuity: At 12 months of follow up in our study eyes there was a statistically significant change in the BCVA compared to baseline in both the groups. The average gain in visual acuity was however higher in the intravitreal anti-VEGF group.(8.825 ETDRS letters compared with 3.09ETDRS letters in the laser only group at 12 months of follow up,Table 1)

Table 1

\begin{tabular}{|c|c|c|c|c|}
\hline & $\begin{array}{l}\text { Group } 1 \\
(\text { Mean } \pm \text { SD })\end{array}(\mathbf{N}=40)$ & $\begin{array}{l}\text { Group } 2 \\
(\text { Mean } \pm \text { SD })\end{array}(\mathrm{N}=40)$ & $\begin{array}{l}\text { Total } \\
(\text { Mean } \pm \text { SD })\end{array}$ & p-value \\
\hline \multicolumn{5}{|c|}{ BCVA(ETDRS letters) } \\
\hline Baseline & $18.725 \pm 12.21$ & $25.825 \pm 7.76$ & $22.275 \pm 10.77$ & .003 \\
\hline 4 weeks & $27.5 \pm 15.3$ & $29.55 \pm 8.46$ & $28.525 \pm 12.33$ & .461 \\
\hline & & & & \\
\hline
\end{tabular}


Comparison Study Of Efficacyinvolving Intravitreal Ranibizumab Versus....

\begin{tabular}{|l|l|l|l|l|}
\hline 6months & $28.05 \pm 12.98$ & $28.7 \pm 9.24$ & $28.375 \pm 11.2$ & .797 \\
\hline 12 months & $27.1 \pm 13.39$ & $28.5 \pm 8.62$ & $27.8 \pm 11.21$ & .580 \\
\hline
\end{tabular}

It was also noted that maximum improvement in visual acuity in the laser group was during the period of first 4 weeks with slight decline thereafter. While for the ivR group maximum improvement was noted during the first 4 weeks to 6 months which reduced subsequently at 12 months of follow up (though remaining statistically higher than the baseline and also higher than that in the laser only group through 12 months of follow up).

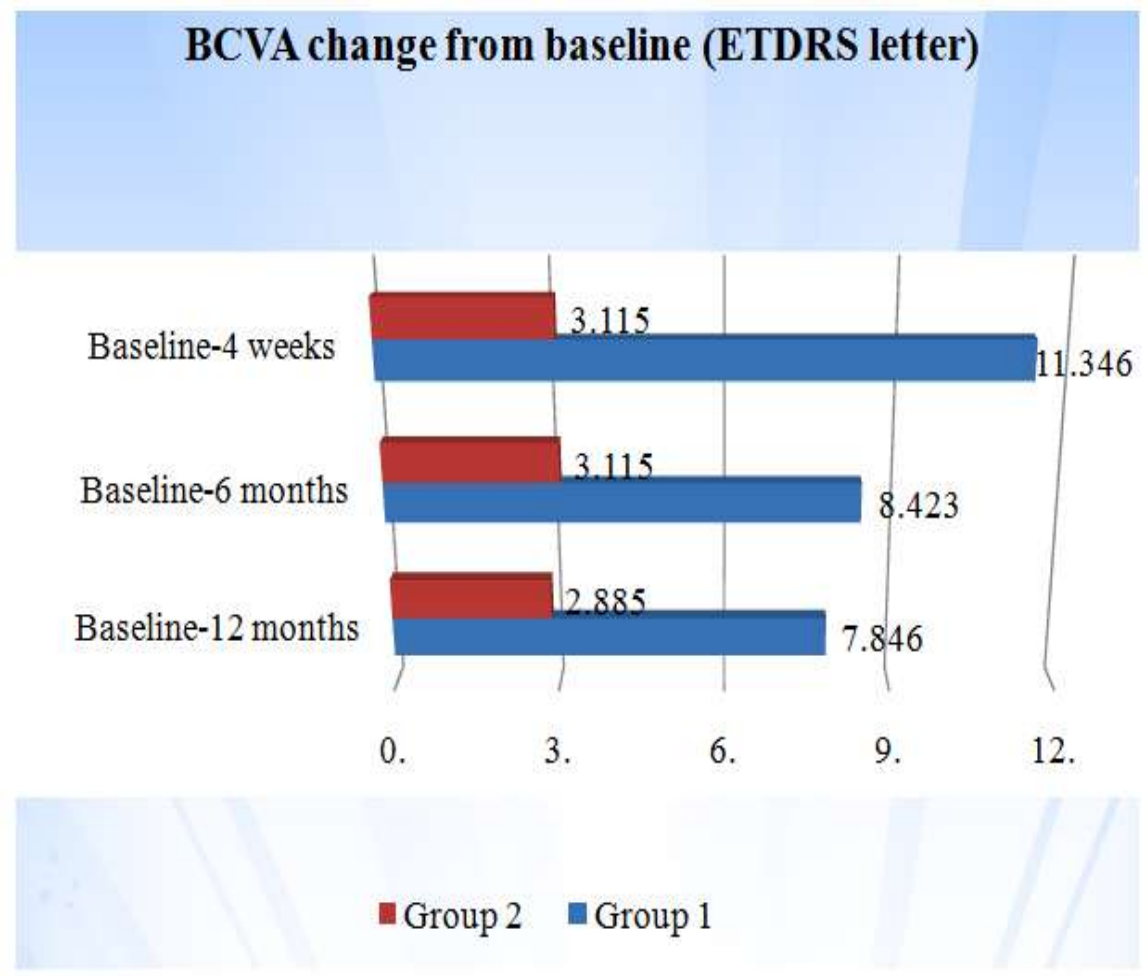

This can be explained by the effectiveness of initial use of anti-VEGF for rapidly reducing edema and hence improving visual acuity in this group and also by the fact that the maximal effect of the intravitreal antiVEGF injections is observed to last during the first $2-4$ weeks. At $6^{\text {th }}$ month of follow up there was significant difference between the two groups in visual acuity change which could not be attributed to chance alone as signified by the $\mathrm{p}$ value $<0.05$.

Effect on Central macular thickness: At 12th month of follow up in our study eyes there was a significant change in the CMT compared to baseline in both the groups. The decrease from the average baseline CMT was higher in the intravitreal anti-VEGF group (74.22microns compared with 28.54 microns in the laser only group at 12 months follow up,Table 2)

Effect of treatment on Central Macular Thickness(Table 2)

\begin{tabular}{|c|c|c|c|c|}
\hline CMT & $\begin{array}{l}\text { Group I } \quad(N=40) \\
(\text { Mean } \pm \text { SD) }\end{array}$ & Group $2(\mathrm{~N}=40)($ Mean \pm SD $)$ & $\begin{array}{l}\text { Total } \\
(\text { Mean } \pm \text { SD) }\end{array}$ & p-value \\
\hline Baseline & $403.17 \pm 151.19$ & $333.575 \pm 80.08$ & $368.38 \pm 125.09$ & $0.012 \mathrm{~S}$ \\
\hline 4 weeks & $313.27 \pm 87.87$ & $302.875 \pm 87.85$ & $308.075 \pm 87.46$ & $0.59 \mathrm{NS}$ \\
\hline 6months & $325.65 \pm 98.09$ & $305.5 \pm 91.2$ & $315.575 \pm 94.646$ & $0.34 \mathrm{NS}$ \\
\hline
\end{tabular}




\begin{tabular}{|l|l|l|l|l|}
\hline 12 months & $347.925 \pm 106.78$ & $306.725 \pm 74.6$ & $327.325 \pm 93.84$ & $0.049 \mathrm{~S}$ \\
\hline
\end{tabular}

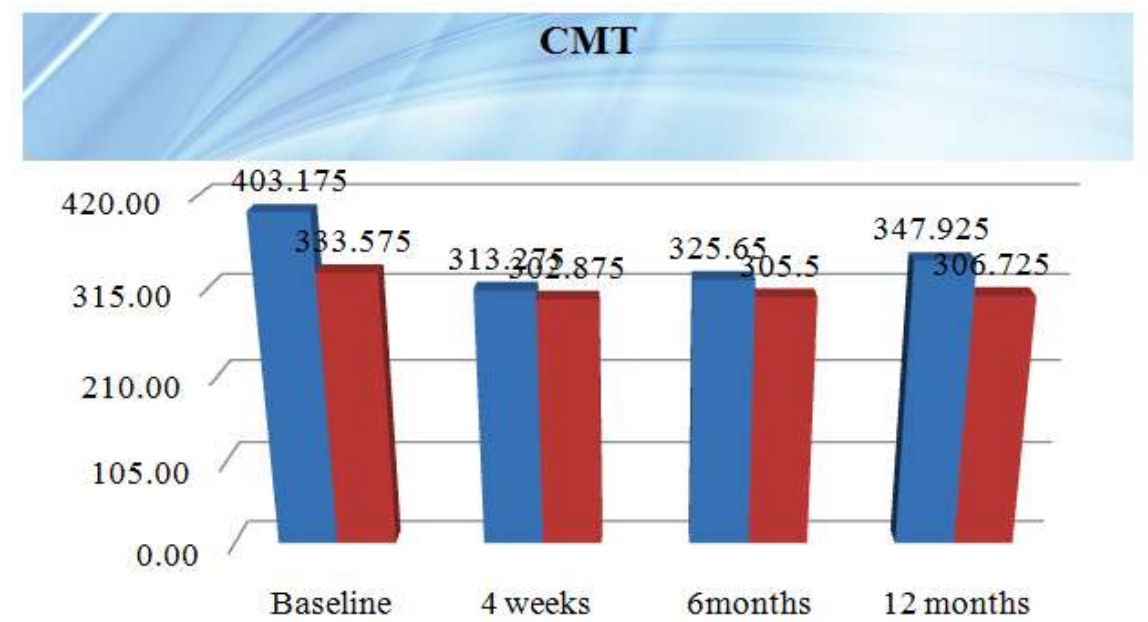

Group 1 Group 2

\section{Discussion}

The purpose of the study was to evaluate the effect of macular photocoagulation in DME, to evaluate the effect of intravitreal anti-VEGF (Ranibizumab) inDME, and finally to compare the effect of the established mode of therapy that is Modified macular photocoagulation alone with intravitreal anti-VEGF in DME. This was a prospective, randomized interventional study of 80 eyes of 80 patients with DME involving the fovea. The patients recruited were all > 18 yrs of age with a mean age of 58.69 years. There was a 63.9 percent male preponderance with respect to eyes recruited in the study. The patients selected on the basis of inclusion and exclusion criteria were divided into two groups by randomization. Group 1 was subjected to anti-VEGF therapy. Group 2 was subjected to modified macular photocoagulation. The main outcome parameters were BCVA change (ETDRS letters) at 12th month of follow up and CMT change (microns) at 12th month of follow.

There was a statistically significant change in both the groups in BCVA (ETDRS letters) at 4 weeks, 6 months and 12 months of follow up compared to the baseline. Group 1 comprising of intravitreal anti-VEGF however showed a tendency towards greater increase, mean gain in BCVA being more in this group. It was noted by intergroup analysis also that there was a significant difference between the two groups in BCVA change at 12 months which could not be attributed to chance alone. There was significant decrease in the CMT from baseline in the laser group only at 12 months of follow up. The intravitreal anti-VEGF group showed significant decrease in the CMT at 4 weeks, 6 months and 12 months of follow up. The intravitreal anti-VEGF group tended to show greater mean reduction in CMT amongst the two groups at all the follow up intervals through 6 months. . It was noted by intergroup analysis also that there was a significant difference between the two groups in CMT change at 12 months which could not be attributed to chance alone. There were no systemic or severe ocular side effects in the study eyes. No cases of endophthalmitis or persistently elevated intraocular pressures occurred in the study eyes.

Both the interventional methods were effective in improving visual acuity. On an average the improvement in group 1 (anti-VEGF) was 8.825 ETDRS letters as compared to 3.09 in group 2 (laser only) over the entire duration of study i.e. 12 months showing the greater effectiveness of the group 1 intervention. It was also seen that maximum improvement in visual acuity in the laser group was during the period of first 4 weeks then slight decline thereafter. While for the intravitreal anti VEGF maximum improvement was noted during the first 4 weeks which was maintained till 6months, which reduced subsequently at 12 months of follow up (though remaining higher than the baseline and also higher than that in the laser only group through the 12 months of follow up). 
The limitations of our study include the small number of patients and relatively short follow up. Also the greater visual acuity gain in the combined anti-VEGF could be to some extent attributed to the initial worse mean visual acuity in this group compared to the laser only group. In a trial by DRCR.net to identify factors associated with the visual acuity outcome following focal/grid photocoagulation for DME it was reported that the likelihood of improving 10 or more letters ( 2 or more lines) was greater when baseline visual acuity was poor and the likelihood of worsening 10 or more letters was greater when baseline visual acuity was good. This likely reflected, at least in part, effects on the amount of improvement that can occur when acuity is only mildly reduced and the amount of worsening that can occur when visual acuity is poor.

\section{Conclusion}

Both macular photocoagulation alone and intravitreal Ranibizumab were effective in the treatment of DME in terms of visual acuity gain and CMT reduction on OCT. Intravitreal Ranibizumab however was more effective than macular photocoagulation alone in improving visual prognosis in patients of DME at 12 months of follow up, without causing significant systemic or ocular side effects. Intravitreal anti-VEGF alsolead to greater reduction in CMT compared to macular photocoagulation alone.It was noted by intergroup analysis alsothat there was a significant difference between the two groups in BCVA change at 12 months which could not be attributed to chance alone.

\section{References}

1. MossSE,KleinR,KleinBE.en-yearincidenceofvisualloss in a diabetic population. Ophthalmology 1994;101:1061-70.

2. Paulus YM, Gariano RF. Diabetic retinopathy: a growing concern in an ageing population.Geriatrics 2009;64:16-20

3. Klein R, Klein BE, Moss SE, Davis MD, DeMets DL. The Wisconsin Epidemiologic Study of Diabetic Retinopathy. II. Prevalence and risk of diabetic retinopathy when age at diagnosis is less than 30 years. ArGh Ophthalmology 1984; 102:520-526.

4. King H, Aubert RE, Herman WH. Global burden of diabetes, 1995-2025 prevalence, Numerical Estimates and Projection. Diabetes Care 1998; 1414:31.

5. Deepa M, Pradeepa R, Rema M, Mohan A, Deepa R, Shanthirani S, Mohan V. The Chennai Urban Rural Epidemiology Study study design and methodology (urban component) (CURES-I). J Assoc Physicians India 2003 Sep;51 :863-70.

6. Aiello LP, Avery RL, Arrigg PG, et al. Vascular endothelial growth factor in ocular fluid of patients with diabetic retinop- athy and other retinal disorders. N Engl J Med 1994;331: 1480 -

7. Dandona L, Dandona R, John RK. Estimation of blindness in India from 2000 through 2020: implications for the blindness control policy. Natl Med J India 2001;14 (6):327-34.

8. Hellstrom M, Gerhardt H, Kalen M et al. Lack of pericytes leads to endothelial hyperplasia and abnonnal vascular morphogenesis. Journal of Cell Biology 2001; 152 (3):543-553.

9. Lindahl P, Johansson BR, Leveen P, and Betsholtz C. Pericyte loss and microaneurysm formation in PDGF-B deficient mice. Scien e 1997; 277 (5323): 242-245.

10. Preferred practice pattem: diabetic retinopathy. San Francisco (CA). American Academy of Ophthalmology 2003.

11. Moss SE, Klein R, Klein BE. Ten-year incidence of visual loss in a diabetic population. Ophthalmology 1994; 101 (6): 1061-1070.

12. Romero-Aroca P, Femandez-Balart J, Baget-Bemaldiz M, Martinez-Salcedo I, Mendez-Marin I, Salvat-Serra M et al. Changes in the diabetic retinopathy epidemiology after 14 years in a population of Type I and 2 diabetic patients after the new diabetes mellitus diagnosis criteria and a more strict control of the patients. J Diabetes Complications 2009; 23: 229-238.

13. Klein R, Lee KE, Knudtson MD, Gangnon RE, Klein BE. Changes in visual impainnent prevalence by period of diagnosis of diabetes: the Wisconsin Epidemiologic Study of Diabetic Retinopathy. Ophthalmology 2009; 116: 1937-1942.

14. Williams R, Airey M, Baxter H, Forrester J, Kennedy-Martin T, Girach A. Epidemiology of diabetic retinopathy and macular oedema: a systematic review. Eye (Lond) 2004; 18: 963-983.

15. Miljanovic B, Glynn RJ, Nathan DM, Manson JE, Schaumberg DA. A prospective study of serum lipids and risk of diabetic macular edema in type 1 diabetes. Diabetes 2004; 53: 2883-2892.

16. Scholl S, Kirchhof J, Augustin AJ. Pathophysiology of macular edema. Ophthalmologica 201 0; 224 (1 ): 8-15.

17. Barile GR, Pachydaki SI, Tari SR, Lee SE, Donmoyer CM, Ma W et al. The RAGE axis in early diabetic retinopathy. Invest Ophthalmol Vis Sci 2005; 46: 2916-2924. 